\title{
Positronium Diffusion in Polystyrene at Low Temperatures
}

\author{
Kenji Ito and Yusuke UjIHIRA \\ Research Center for Advanced Science and Technology, The University of Tokyo, \\ 4-6-I Komaba, Meguro-ku, Tokyo 153-8904, Japan.
}

(Received October 26, 1997)

\begin{abstract}
Diffusion of ortho-positronium (o-Ps) in polystyrene (PS) at low temperatures (20-300 K) was studied by means of the positron annihilation lifetime technique. 2,2'-Dinitrobiphenyl was added to PS as a Ps quencher, and the diffusion coefficients were determined from the measured Ps quenching rate constants, assuming that the reaction between Ps and the quencher is entirely diffusion-controlled. The determined diffusion coefficient increases with increasing temperature, and is significantly influenced by such structural relaxation of PS as $\delta$ and $\beta$ transitions. The estimated diffusion length of $o$-Ps, $1.5-2.5 \mathrm{~nm}$, is only a few times larger than the Ps cavity size, indicating that the effect of the Ps diffusion on the Ps lifetime distribution is almost insignificant in PS at low temperatures. The size distributions of free volume in pure PS were obtained from the lifetime distributions of $o$-Ps without taking an effect of the diffusivity of $o$-Ps into consideration. Peculiar broadening of the size distribution of free volume for PS, which is induced by an inhomogeneous local structure, is observed at the transition temperatures, $T_{\delta}$ and $T_{\beta}$.

KEY WORDS Polystyrene / Diffusion Coefficient / Free Volume / Positronium / Positron Annihilation / Structural Relaxation /
\end{abstract}

The free volume concept has been extensively used to interpret various macroscopic physical properties ${ }^{1-7}$ of polymers such as viscosity, elasticity, glass transition, and structural relaxation, all of which are important from the view point of controlling mechanical properties of polymers. The data obtained by such microscopic techniques as fluorescence, ${ }^{8}$ photochemical hole burning, ${ }^{9}$ and nuclear magnetic resonance ${ }^{10}$ are often discussed in relation to the predictions of the free volume theory. Another empirical technique, which can sensitively probe the local structure of the polymers, is positron annihilation.

The observed decay curve of positrons annihilating in a polymer contains a long-lived component with an average lifetime of $1-5 \mathrm{~ns}$. The component is attributed to "pick-off" annihilation of ortho-positronium (o-Ps: the spin-parallel bound state between a positron and an electron) localized in a sub-nanometer size hole. A relation between the pick-off annihilation lifetime and the cavity size was derived based on a simple quantum mechanical model initially proposed by $\mathrm{TaO}^{11}$ and later developed by Eldrup et al., ${ }^{12}$ and Nakanishi et al. ${ }^{13,14}$ Several empirical correlations between the average Ps cavity size estimated from the relation and free volume properties of polymers were reported. ${ }^{15,16}$ In addition to this, attempts have been made to deduce the size distribution of the Ps cavity by inverse Laplace transformation of the positron lifetime data.

This paper concerns diffusion of Ps in a polymer at low temperatures. Following Hirata et al. ${ }^{17}$ and Kobayashi et al., ${ }^{18}$ 2,2'-dinitrobiphenyl (DNB) was added to polystyrene (PS) as a Ps quencher, and the diffusion coefficients were determined from the measured Ps quenching rate constants, assuming that the reaction between Ps and the quencher is entirely diffusioncontrolled. The diffusion coefficient in PS was found to be affected by the local molecular motion as in the case of polycarbonate (PC). ${ }^{18}$ The estimated diffusion length of $1.5-2.3 \mathrm{~nm}$ is only a few times larger than the Ps cavity size, and it is concluded that the effect of the Ps diffusion on the Ps lifetime distribution is almost insignificant in PS at low temperatures.

\section{EXPERIMENTAL}

\section{Polystyrene Sample}

The PS sample was obtained from Scientific Polymer Products, Inc. The molecular weight was 190000 . The samples were dissolved, together with six different amounts of DNB ranging from 0 to $2.9 \mathrm{wt} \%$, in dechloromethane solutions, which were then cast onto glass plates. The transparent films thus obtained were dried at an elevated temperature under vacuum until constant weights were obtained.

\section{Positron Annihilation Lifetime Measurement}

Decay curves of positron annihilation were recorded with a conventional fast-fast coincident system employing $\mathrm{BaF}_{2}$ scintillators. The overall time resolution of the system was $\sim 290 \mathrm{ps}$ FWHM (full width at half maximum). ${ }^{19}$ The positron source was $7.5 \times 10^{5} \mathrm{~Bq}$ ${ }^{22} \mathrm{NaCl}$ sealed between Kapton foils with a thickness of $7.5 \mu \mathrm{m}$. The measurements were performed under a vacuum of $\sim 10^{-3} \mathrm{~Pa}$. The samples were cooled down by using a thermostat (Cryo Mini, Iwatani) to $20 \mathrm{~K}$. The lifetime data were recorded with increasing temperatures up to $300 \mathrm{~K}$. Each decay curve was accumulated for more than $4.5 \mathrm{~h}$ and stored in a personal computer. After each measurement the sample temperature was raised to a next value with a heating rate of $\sim 1.5 \mathrm{~K}$ $\min ^{-1}$.

\section{Data Analysis}

Obtained decay curves with total counts of $1-4 \times 10^{6}$ were fitted by a non-linear least-square method. The decay curves were decomposed into three components, a short-lived $\tau_{1}$, an intermediate-lived $\tau_{2}$, and the longest-lived $\tau_{3}$ by using the PATFIT- $88^{20}$ computer program. In the process of the data analysis, the lifetime of para-positronium ( $p$-Ps), $\tau_{1}$, was fixed to its 
intrinsic lifetime of $125 \mathrm{ps}$ and the intensity, $I_{1}$, was assumed to be one third of the $o$-Ps yield, $I_{3}$. The average values of $\tau_{3}$ thus obtained were $7.6 \pm 1.1 \%$ smaller than those obtained without restraints. The obtained lifetimes of $\tau_{3}$ were in the range from $1.7 \mathrm{~ns}$ to $2.0 \mathrm{~ns}$.

Inverse Laplace transformation of positron annihilation decay curve accumulating $4-8$ million counts were performed using the CONTIN program ${ }^{21,22}$ to get lifetime probability density functions (PDFs). The process to obtain the annihilation lifetime distribution of $o$-Ps in polymers has been discussed in previous papers. ${ }^{23-25}$

A lifetime component with an average lifetime longer than $\sim 1 \mathrm{~ns}$ is attributed to the pick-off annihilation of $o$-Ps. The relationship between the observed lifetime of $o$-Ps, $\tau_{3}$, and the cavity radius, $R$, is given by the following semiempirical expression derived from the quantum mechanical model. ${ }^{11-14}$

$$
\tau_{3}=0.5\left[1-\frac{R}{R+\Delta R}+\frac{1}{2 \pi} \sin \left(\frac{2 \pi R}{R+\Delta R}\right)\right]^{-1}
$$

where $\Delta R$ is the thickness of an electron layer on the cavity wall, whose value of $0.166 \mathrm{~nm}$ is known to reproduce known cavity sizes in zeolites and other substances. ${ }^{14}$ The validity of the expression for organic polymers was confirmed by a comparison of the cavity radius, obtained under the assumption of $\Delta R=0.166 \mathrm{~nm}$, with the free volume deduced by the group contribution method of Bondi. ${ }^{15,26}$

\section{RESULTS AND DISCUSSION}

\section{Average Cavity Size in Pure PS}

The average $o$-Ps lifetimes observed in pure PS were converted to the Ps cavity radii by using eq 1 . The average cavity size thus determined is shown in Figure 1 as a function of temperature. The cavity radius increases from 0.26 to $0.29 \mathrm{~nm}$ over the temperature range studied, in agreement with the previous result by $\mathrm{Li}$ et al. ${ }^{27}$ The temperature dependence of the cavity size has clear transitions at $\sim 70$ and $220 \mathrm{~K}$. Based on the available data by dielectric measurements at frequencies of $1 \mathrm{~Hz}$ $(220 \mathrm{~K})$ and $7 \times 10^{3} \mathrm{~Hz}(\sim 70 \mathrm{~K}),{ }^{28}$ we attribute these transitions to $\delta(\sim 70 \mathrm{~K})$ and $\beta(220 \mathrm{~K})$ relaxation of PS. The temperature coefficient of the free volume cavity radius shows a twofold increase at $\sim 220 \mathrm{~K}$, implying that the free volume cavity radius in PS is strongly influenced by the structural change induced by $\beta$ relaxation.

Temperature Dependence of Diffusion Coefficient of o-Ps As shown in Figure 2, the $o$-Ps annihilation rate, $\lambda_{3}$ $\left(=1 / \tau_{3}\right)$, observed in the samples containing from 0 up to $2.9 \mathrm{wt} \% \mathrm{DNB}$, increased with increasing quencher concentration, following the simple kinetic equation,

$$
\lambda_{3}=\left(\tau_{3}^{0}\right)^{-1}+k^{\prime} C
$$

where $\tau_{3}^{0}$ is the lifetime of $o$-Ps in PS containing no DNB, $k^{\prime}$ is the Ps quenching rate constant in units of $(\mathrm{wt} \%)^{-1}$ $\mathrm{ns}^{-1}$ and $C$ is the DNB concentration in wt $\%$. The Ps quenching rate constant in units of $\mathrm{dm}^{3} \mathrm{~mol}^{-1} \mathrm{~ns}^{-1}$ is written as $k=(M / 10 \rho) k^{\prime}$, where $M$ and $\rho$ are the molecular weight of DNB (=244.21) and the density of PS in $\mathrm{g} \mathrm{cm}^{-3}$, respectively. By assuming that the reac-

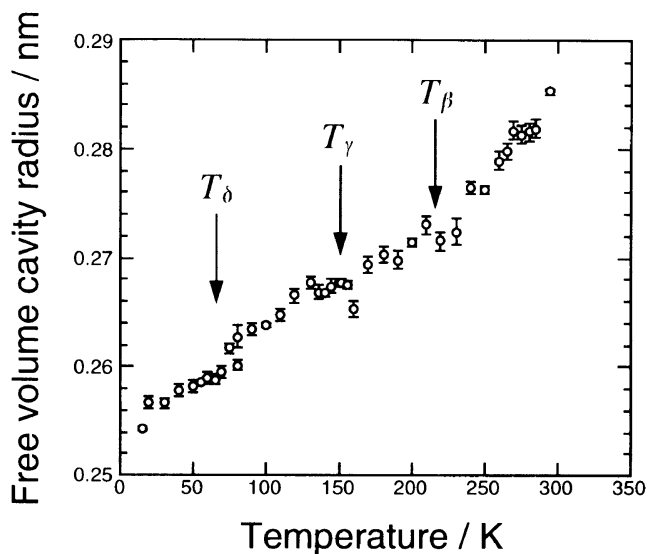

Figure 1. Temperature dependence of the average free volume cavity radius.

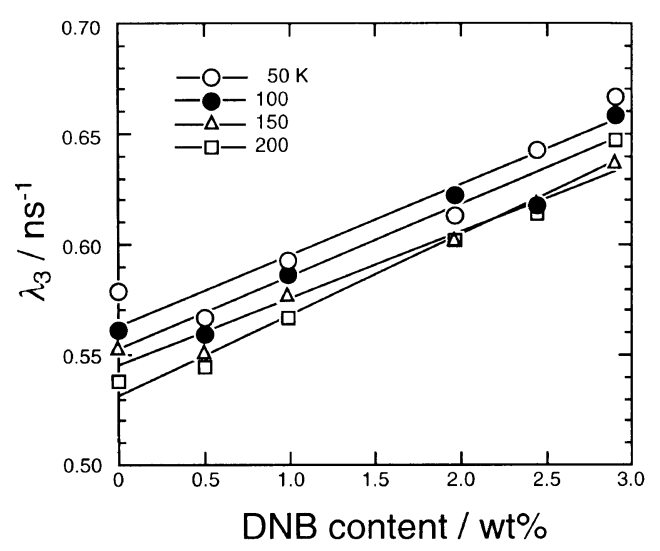

Figure 2. Dependencies of $o$-Ps annihilation rate, $\lambda_{3}$, on addtion of DNB to PS at several temperatures.

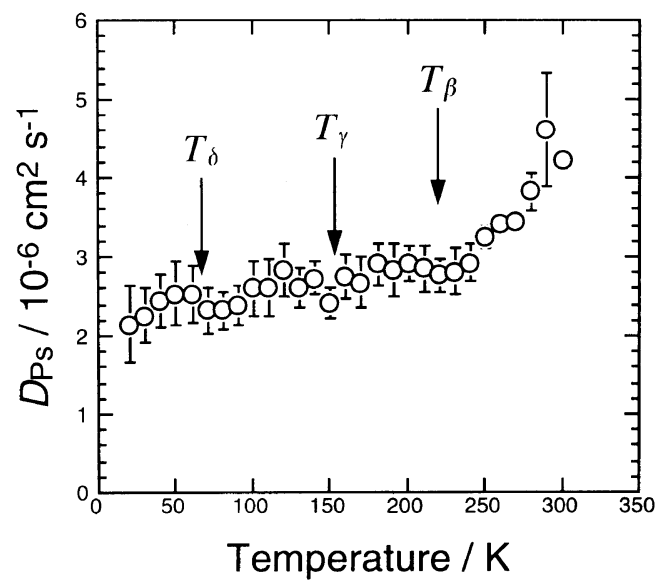

Figure 3. Temperature dependence of the diffusion coefficient of $o$-Ps in PS.

tion between Ps and DNB is diffusion-controlled according to Hirata et al., diffusion coefficients of $o$-Ps, $D_{\mathrm{Ps}_{\mathrm{s}}}$, in PS at various temperatures were determined by the following expression, ${ }^{17}$

$$
D_{\mathrm{Ps}}=6.5 \times 10^{18} k /\left(\pi N_{\mathrm{A}}\right)
$$

where $N_{\mathrm{A}}$ is the Avogadro constant. Results are shown in Figure 3.

It is seen in Figure 3 that peculiar changes of the temperature dependence of the diffusion coefficient are 
observed at $\sim 60 \mathrm{~K}$ and $\sim 220 \mathrm{~K}$, which are close to the transition temperatures of $\delta$ and $\beta$ relaxation of PS. There may be slight change of the diffusion coefficient at $150 \mathrm{~K}$ corresponding to $\gamma$ relaxation measured at a frequency of $1 \mathrm{~Hz}^{28}$ as well. These observations suggest that the diffusion coefficient of Ps sensitively probes local motions of the main and side chains of PS. It is also seen that the temperature dependence of the diffusion coefficient is somewhat related to the variation of the Ps cavity size in Figure 1, an indication that the diffusion process of Ps may be affected by a change in the free volume.

As is seen from Figure 4 , where $\ln \left(D_{\mathrm{Ps}}\right)$ is plotted $v s$. reciprocal temperature, variation of the diffusion coefficient with temperature is characterized by two regions, which are clearly separated by $\beta$ transition. The temperature dependence of $D_{\mathrm{Ps}}$ in each region approx-

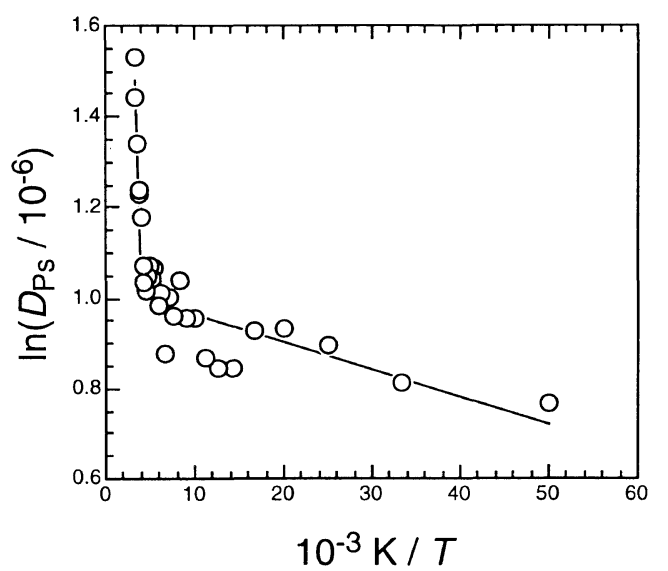

Figure 4. Logarithmic diffusion coefficient versus reciprocal temperature for PS. imately follows the Arrhenius equation and activation energies determined from the solid lines of Figure 4 are listed in Table I, together with the activation energies in polysulfone (PSF) and PC above room temperature, which were estimated based on the diffusion data reported by Hirata et $a .^{17}$ In this table, the activation energies of the diffusion for several gas molecules such as $\mathrm{O}_{2}, \mathrm{~N}_{2}$, and $\mathrm{Ar}{ }^{29}$ in the glassy state of polycarbonate (tetramethyl bisphenol A) (TMPC), are also included. Below $T_{\beta}$ the activation energy of $o$-Ps in PS is very small and only $0.012 \mathrm{kcal} \mathrm{mol}^{-1}$. Above $T_{\beta}$ the activation energy is higher $\left(0.82 \mathrm{kcal} \mathrm{mol}^{-1}\right)$ and close to the values for PSF and PC, suggesting common nature of the Ps diffusion process in polymers at high temperatures. However, the activation energy compared with about $1 \mathrm{kcal} \mathrm{mol}^{-1}$ is much smaller than the activation energies for the gas molecules. In light of the extremely small mass of Ps, the lower activation energies may indicate that tunneling of Ps plays an important role in the diffusion of Ps. The possibility of Ps tunneling was suggested by $\mathrm{Yu}$ et al $^{30}$ and other authors. ${ }^{31,32}$ Proper incorporation of the quantum mechanical model on Ps tunneling into our diffusion model, however, may be subject of the further investigation.

Substituting of $D_{\mathrm{Ps}_{\mathrm{s}}}$ in Figure 3 and $o$-Ps lifetimes of $1.7-2.0 \mathrm{~ns}$ in, ${ }^{33}$

$$
l_{\mathrm{Ps}}=\sqrt{6 D_{\mathrm{Ps}} \tau_{3}}
$$

results in the average diffusion length, $l_{\mathrm{Ps}}$, of 1.5 to $2.3 \mathrm{~nm}$, which are only a few times larger than the Ps cavity sizes in Figure 1. This reveals that the lifetime distribution of $o$-Ps atoms is only a little affected by the diffusion effect. Because obtained diffusion length of 1.5 to 2.3

Table I. Apparent activation energies for diffusion of $o$-Ps and some gas molecules in polymers

(in the glassy state)

${ }^{a}$ These values were estimated based on the diffusion data reported by Hirata et al. ${ }^{17}{ }^{b}$ In this study. The data of an apparent activation energy of diffusion were obtained by such respective gas molecules as $* \mathrm{O}_{2},{ }^{* *} \mathrm{Ar},{ }^{* * *} \mathrm{~N}_{2}{ }^{28}$ 


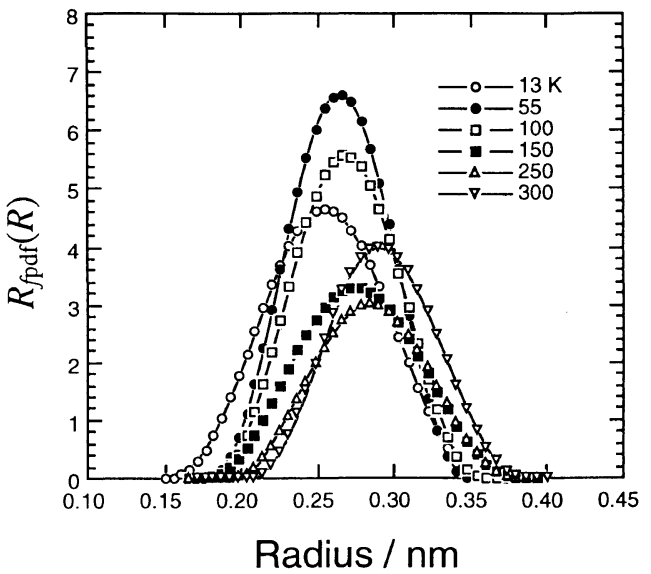

Figure 5. Size distribution of free volume in PS at several temperatures.

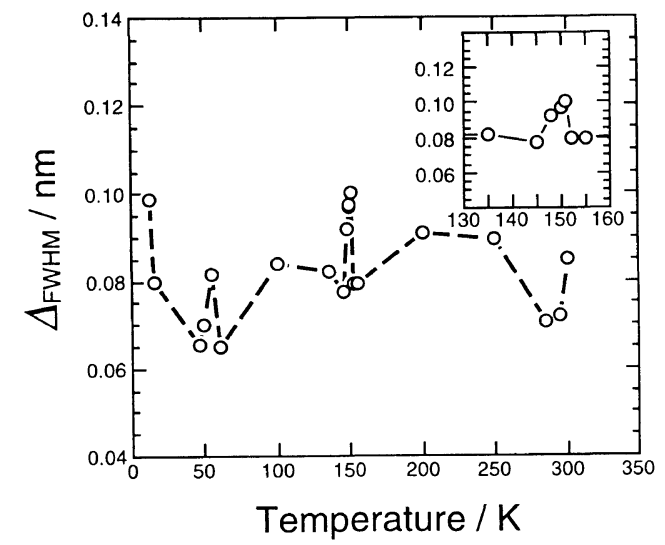

Figure 6. Temperature dependence of the FWHM (full width at half maximum) of free volume size distribution of PS. FWHM is a measure of the inhomogeneity of the nanoscopic structure.

$\mathrm{nm}$ is almost equal to the distance between the center of one free volume hole and that of the nearest-neighbor hole $(1.75 \mathrm{~nm})$, according to $\mathrm{Yu}$ et al. ${ }^{30}$ This suggests that $P$ s is trapped in an approximate single free volume during the entire lifetime, therefore, Ps diffusion plays only a minor role in determing the distribution of $o$-Ps lifetimes in pure PS at low temperatures.

\section{Size Distribution of Ps Cavities in Pure PS}

It is possible to discuss size distribution of Ps cavities based on the lifetime distribution analyzed by the CONTIN program. For simplicity, we assume that the effect of Ps diffusion is completely negligible in pure PS. Then the cavity size distribution is calculated from the probability density function of the annihilation rate, $\alpha(\lambda)$, according to the following equation,

$$
\begin{aligned}
R_{f \mathrm{pdf}}(R) & =-\alpha(\lambda) \frac{\mathrm{d} \lambda}{\mathrm{d} R} \\
& =-0.331\left[\cos \left(\frac{2 \pi R}{R+0.166}\right)-1\right] \frac{\alpha(\lambda)}{(R+0.166)^{2}}
\end{aligned}
$$

Some of the cavity radius distributions thus obtained are shown in Figure 5. With increasing temperature, the peak position of the distribution is shifted toward a larger radius, as is the average cavity radius in Figure 1. Cavity size distribution deduced from position lifetime data may reflect the fluctuation of the free volume induced by local motions of the polymer chain. FWHM

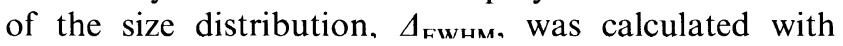
an error range of $\pm \sim 2 \%$ as a measure of the inhomogeneity of the nanoscopic structure, and its temperature dependence is presented in Figure 6.

The $\Delta_{\mathrm{FWHM}}$ exhibits pronounced maxima at $T_{\delta}$ and $T_{\beta}$. Similar maxima were observed for high molecular weight PS at $\alpha$ and $\beta$ transitions by Li et al. ${ }^{27}$ Thus it seems that the structural transition of PS requires a fairly large change of the nanoscopic free volume and the structure of PS at a transition temperature is highly inhomogeneous. The significant increases of the $\Delta_{\mathrm{FWHM}}$ of the free volume cavity distribution at structural transitions of PS may reveals that entanglement of the molecular chains plays an important role in the structural transition of PS.

\section{CONCLUSION}

The diffusion coefficient of Ps in PS was determined at low temperatures by making use of Ps quenching by DNB. Temperature dependence of the determined diffusion coefficient was well described by the Arrhenius equation, with different activation energies below and above $T_{\beta}$. The average diffusion length of Ps was found to be $1.5-2.3 \mathrm{~nm}$, only a few times larger than the Ps cavity radius, and it was concluded that the effect of Ps diffusion on the Ps lifetime distribution is almost insignificant.

By assuming that the effect of the Ps diffusion is completely negligible, cavity size distribution in pure PS was obtained from the lifetime distribution analyzed by the CONTIN program. Temperature dependence of the free volume cavity distribution revealed that the distribution is anomalously broadened at $T_{\delta}$ and $T_{\beta}$, suggesting that the structural transition of Ps requires a fairly large free space, and that the structure of PS at the transition temperature is highly inhomogeneous.

Acknowledgment. The authors are grateful to Dr. Yoshinori Kobayashi and Dr. Koichi Hirata (National Institute of Materials and Chemical Research) for fruitful and stimulating discussions.

\section{REFERENCES AND NOTES}

1. P. J. Flory, J. Chem. Phys., 10, 51 (1942).

2. M. L. Huggins, J. Phys. Chem., 46, 151 (1942).

3. A. K. Doolittle, J. Appl. Phys., 22, 1471 (1951).

4. M. L. Williams, R. F. Landel, and J. D. Ferry, J. Am. Chem. Soc., 77, 3701 (1955).

5. R. Simha and R. F. Boyer, J. Chem. Phys., 37, 1003 (1962).

6. R. Simha and T. Somcynsky, Macromolecules, 2, 342 (1969).

7. R. E. Robertson, R. Simha, and J. G. Curro, Macromolecules, 18, 2239 (1985).

8. E. F. Meyer, A. M. Jamieson, and R. Simha, Polymer, 31, 243 (1990).

9. S. Tanaka, S. Machida, T. Yamashita, and K. Horie, Macromol. Chem. Phys., 197, 4095 (1996).

10. T. T. P. Cheung and P. J. Chu, J. Phys. Chem., 96, 9551 (1992).

11. S. J. Tao, J. Chem. Phys., 56, 5499 (1972).

12. M. Eldrup, D. Lightbody, and J. N. Sherwood, Chem. Phys., 63, 51 (1981).

13. H. Nakanishi, S. J. Wang, and Y. C. Jean, in "Positron 
Annihilation Studies of Fluids," S. C. Sharma, Ed., World Scientific, Singapore, 1988, p 292.

14. H. Nakanishi, Y. C. Jean, in "Positron and Positronium Chemistry," D. M. Schrader and Y. C. Jean, Ed., Elsevier, Amsterdam, 1988, p 159.

15. Y. Kobayashi, K. Haraya, Y. Kamiya, and S. Hattori, Bull. Chem. Soc. Jpn., 65, 160 (1992).

16. Y. Kobayashi, K. Haraya, S. Hattori, and T. Sasuga, Polymer, 35, 925 (1994)

17. K. Hirata, Y. Kobayashi, and Y. Ujihira, J. Chem. Soc., Faraday Trans., 92, 985 (1996).

18. Y. Kobayashi, K. Hirata, Y. Ujihira, and K. Ito, J. Radioanal. Nucl. Chem., Articles, 210, 525 (1996).

19. N. Furusaki, Y. Ohko, and Y. Ujihira, Kobunshi Ronbunshu, 50, 969 (1993).

20. P. Kirkegaard, N. J. Pederson, and M. Eldrap, PATFIT-88: A Data-Processing System for Positron Annihilation Spectra on Mainframe and Personal Computers, Tech. Rep. Risø-M-2740 (Risø National Laboratory, DK-4000 Roskilde, Denmark 1989).

21. S. W. Provencher, CONTIN (Veresion 2) Users Manual, Tech. Rep. EMBL-DA07 (Max-Planck-Institut Für Biophysikalische
Chemie, Göttingen, 1984).

22. R. B. Gregory, CONTIN (PAL-2): A modification of CONTIN (Version 2) for the Determination of Positron Annihilation Lifetime Distributions (Kent State University, Kent, OH, 1993).

23. R. B. Gregory and A. Procyk, ref 13, p 524.

24. R. B. Gregory, J. Appl. Phys., 70, 4665 (1991)

25. G. H. Dai and Y. C. Jean, Nucl. Instr. Meth. in Phys. Res. B, 99, 357 (1995)

26. A. Bondi, J. Phys. Chem., 68, 441 (1964)

27. H-L. Li, Y. Ujihira, and A. Nanasawa, Kobunshi Ronbunshu, 53 , 358 (1996)

28. R. F. Boyer, H. Keskkula, and A. E. Platt Ed., "Encyclopedia of Polymer Science and Technology," Vol. 13, 1970, p 283.

29. K. Haraya and S-T. Hwang, J. Membrane Sci., 71, 13 (1992).

30. Z. Yu, J. D. McGervey, A. M. Jamiesom, and R. Simha, Macromolecules, 28, 6268 (1995).

31. Y. C. Jean, Macromolecules, 29, 5756 (1996).

32. A. H. Baugher, W. J. Kossler, and K. G. Petzinger, Macromolecules, 29, 7280 (1996).

33. S. J. Wang and Y. C. Jean, ref 14, p 259. 